\title{
PIV MEASUREMENTS OF PARTICLE VELOCITIES AND TRAJECTORIES FOR INTERNAL WAVES PROPAGATING IN A TWO-LAYER FLUID ON A SLOPING BOUNDARY
}

\author{
Motohiko Umeyama $^{1}$ and Kim-Cuong Nguyen ${ }^{1}$
}

\begin{abstract}
Some characteristics of internal waves propagating in a two-layer fluid on a sloping bottom were investigated experimentally using particle image velocimetry (PIV) that was originally developed to express the velocity field in a two-dimensional area. Velocity distributions were estimated at several phases during one wave cycle. The method of characteristics was adapted to calculate the interfacial displacement and water particle trajectory. In addition, the variations of wave speed, wave height and wave setup from deepwater to shallow-water regions by an imaging technique were compared with those by a wave dissipation model based on the radiation stress concept. These attempts proved the ability of the imaging technique to accurately measure both temporal and spatial variations of some physical quantities due to the propagation of internal waves. The PIV technique was applied to the prediction of Lagrangian velocity in a Eulerian scheme. The measured particle path was compared with the positions found theoretically by the method of characteristics.
\end{abstract}

Keywords: Internal waves; Velocity; Trajectory; Flow measurement; PIV

\section{INTRODUCTION}

Numerous experiments have been carried out to investigate the shoaling and breaking of internal waves on various slopes fabricated in hydraulic laboratories. Thorpe (1966) examined the breaking and runup of internal waves propagating between two density-stratified layers. Close to a point of contact between the density interface and the bottom slope, internal waves steepen at the front as the lower layer becomes shallow, but the crests break backwards. On the upper slope, the wave becomes a blob, and the dense fluid returns down the slope like a density current. Finally, the head-on collision occurs between the backflow and the incident waves. Wunsch (1971) considered the second-order mass and momentum flux carried by the internal waves that encounter a shoaling region, and the wave setup and set-down of the mean density interface caused by the Stokes drift. Using electrical conductivity meters and a shadowgraph technique, Cacchione and Wunsch (1974) performed a series of laboratory experiments for internal waves in a continuously stratified fluid over a uniform slope, and found the interfacial mixing resulting from the wave breaking and the energy dissipation resulting from the bottom viscous. They divided the wave categories into the subcritical and supercritical cases that depend upon the ratio of the bottom slope to the wave-characteristic slope. To investigate the evolution and breaking of an internal wave as it shoals on a sloping bottom connecting the deeper region, Kao et al. (1985) measured the fluid velocity during the passage of internal waves using hot-film anemometers at some fixed locations on the undisturbed pycnocline, and the instantaneous velocity profile using a hydrogenbubble wiring system consisting of platinum wire, high-intensity light source, and slit-light box. They found that the major energy was dissipated over the slope, but the remainder was transmitted onto the shelf or reflected back to the deep-water region. Wallace and Wilkinson (1988) experimentally resolved the internal-wave structure of the breaking process using conductivity probes from which the density field could be evaluated, and investigated the shoaling, mixing and run-up using various visualization techniques which include fraction screen, dye injection and particle tracking methods. Helfrich (1992) observed the interaction of an internal wave of depression with a sloping bottom using micro-scale conductivity-temperature probes, and recognized the importance of the backflow that produces significant mixing. Side-view photographs and video recordings of shadowgraphs and dye movement were also used to obtain quantitative information on the kinematics of breaking and runup. DeSilva et al. (1997) investigated the temporal and spatial density and velocity, boundary-layer extent, turbulent, mixing and intrusion due to the internal wave breaking on a sloping bed, by employing three different flow visualization methods as a rainbow color schlieren method, a digital particle tracking method, and a dye visualization method. The mechanism of internal waves in a two-layer system comprising homogeneous fluids of slightly different densities has been studied since the work of Umeyama (2002) who used a digital video camera to illustrate the internal waves propagating in a fluid of finite depth over a flat bed. After analysing continuous photos, the temporal and spatial variations of the density interface were obtained from a set of luminance values in the upper layer and lower layer, in which the

\footnotetext{
${ }^{1}$ Department of Civil \& Environmental Engineering, Tokyo Metropolitan University, 1-1 Minami-ohsawa, Hachioji, Tokyo 192-0397, Japan
} 
water was visualized by adding a blue dye. To observe the runup and breaking of internal waves over a uniform slope, Umeyama and Shintani (2004) installed a Plexiglas plate in the same wave tank and measured the profile of internal waves and the mixing between two layers. Later, Umeyama and Shintani (2006) performed more precise laboratory tests, by considering additional aspects such as transformation, attenuation, set-down, and setup during the shoaling and breaking events.

Visualization techniques with lasers have played an essential role in fluid flows since the late 1970s, because they yield both qualitative and quantitative insights in fluid mechanics. The developments in particle image velocimetry (PIV) and particle tracking velocimetry (PTV) have led to the visualization of fluid velocity. These two methods have used different evaluation techniques to extract velocity vectors from the images. Especially the progress of PIV in last two decades was huge in its capability of resolving spatial and temporal quantities and wide in its area of the application. The PIV technique is now one of the standard measurement tools for the quantification of the velocity field in fluid mechanics. In contrast, PTV has been developed to measure a sufficient number of velocity vectors with meaningful accuracy. Both techniques are analysis methods for image pairs taken in a seeded flow field with known temporal separation. Michallet and Ivey (1999) measured water velocities due to the shoaling and breaking of internal solitary waves on a uniform slope using PIV. The velocity field was calculated with two consecutive frames by cross-correlation method (Stevens and Coates 1994). Grue et al. (1999) investigated solitary waves propagating in a two-layer fluid using both PIV and PTV. Shimizu et al. (2005) employed a PIV system consisting of a Nd:YAG pulsed laser and a CCD camera, and got precise features of velocity field along a slope during the pass of internal waves. Umeyama and Shinomiya (2009) developed a new PIV system that utilized halogen lamps and three high-definition digital video cameras to expand the field of view. The velocity field was measured under realistic laboratory conditions, and the experimental velocity distributions were compared with the corresponding results by the third-order Stokes internal-wave theory. These attempts proved that the PIV technique enables the measurement of water velocity spatially induced by the nonlinear internal waves with considerable accuracy. Umeyama and Matsuki (2011) recently measured the similar physical quantities with two frequency-doubled Nd:YAG lasers of $50 \mathrm{~mW}$. The PIV method was applied to trace water particle path, and the measured trajectory was compared with the particle positions obtained theoretically by integrating the Eulerian velocity to a higher order in a Taylor series expansion. In addition, the knowledge was extended to analyse mass transport velocity in a shallowwater region.

This article investigates the spatial and temporal variations of the density interface, wave height, celerity and setup due to shoaling and breaking of internal waves, using an image processing technique, and the velocity and trajectory of water particles in an internal wave motion, using PIV. We illustrate 2D instantaneous displacements of density interface from the observed data, and analyse them to obtain the wave height, celerity, and setup. These results are compared with the calculated values by the method of characteristics, the simple shoaling model with energy dissipation, and the momentum balance equation based on a radiation stress concept. The vector fields are presented at several phases in one wave cycle. The PIV technique's ability to measure both temporal and spatial variations of the velocity is proven after a series of attempts. This technique is applied to the prediction of particle trajectory in a Eulerian scheme. The measured particle path is compared to the positions found theoretically by the method of characteristics.

\section{THEORIES}

\section{The method of characteristics for long internal waves on a slope}

Umeyama and Shintani (2004) adapted the method of characteristics, originally developed for the surface waves by Carrier and Greenspan (1957), to express the runup of internal waves at the interface of two fluids of slightly different densities on a sloping bed, and derived a reliable solution for the interfacial displacement and the horizontal velocity. The origin of the axes is located at a point of intersection of the undisturbed density interface and the bed; $x$ is the horizontal coordinate taken to be positive seaward and $z$ is the vertical coordinate pointing the upward. Let denotes the density and thickness of the upper layer $\rho_{I}$ and $h_{I}$, respectively, and those of the lower layer $\rho_{I I}$ and $h_{I I}$, respectively, so that the governing equations are

$$
2\left(1+\frac{\eta_{I I}+h_{I I}}{h_{I}}\right) C_{t}+u_{I I x} C+2\left(1+\frac{\eta_{I I}+h_{I I}}{h_{I}}\right) C_{x}=0
$$




$$
\begin{aligned}
& u_{I I t}+u_{I I} u_{I I x}+2\left(1+\frac{\eta_{I I}+h_{I I}}{h_{I}}\right)^{2} C C_{x}=g \frac{\rho_{I I}-\rho_{I}}{\rho_{I I}} h_{I I x} \\
& C^{2}=g \frac{\rho_{I I}-\rho_{I}}{\rho_{I I}} \frac{\left(\eta_{I I}+h_{I I}\right) h_{I}}{\eta_{I I}+h_{I}+h_{I I}}
\end{aligned}
$$

where $\eta_{I I}(x, t)$ is the vertical displacement of density interface; $u_{I I}(x, z, t)$ is the horizontal velocity; $C$ is the internal wave speed; and $g$ is the gravity acceleration. For simplicity it is assumed the lowerlayer thickness for a uniform slope as

$$
h_{I I}=h_{I I}(x)=m \frac{\rho_{I}}{g\left(\rho_{I I}-\rho_{I}\right)} x
$$

where $m$ is a constant. By adding and subtracting Eqs.(1) and (2), substituting Eq.(4) into them, and assuming $\eta+h_{I I}<h_{I}$, the results can be written in the familiar form:

$$
\left[\frac{\partial}{\partial t}+\left(u_{I I} \pm C\right) \frac{\partial}{\partial x}\right]\left(u_{I I} \pm 2 C-m t\right)=0
$$

The expression in brackets is to be understood as a differential operator. The relation states that the function $\left(u_{I I} \pm 2 C-m t\right)$ is constant for a point moving through the fluid with velocity $\left(u_{I I} \pm C\right)$ for a point whose motion is characterized by the ordinary differential equation $d x / d t=\left(u_{I I} \pm C\right)$. Umeyama and Shintani (2004), using the method of characteristics, made it possible to describe $\eta_{I I}$ and $u_{I I}$ such as

$$
\begin{aligned}
& \eta_{I I}=-\frac{A^{2}}{2} \frac{h_{I}+s x}{h_{I} s x}\left[J_{1}(X) \cos T+\left\{J_{0}(X)-\frac{J_{1}(X)}{X}\right\} \sin T\right]^{2}+A\left\{J_{0}(X) \sin T+J_{1}(X) \cos T\right\} \\
& u_{I I}=A \sqrt{\frac{\left(\rho_{I I}-\rho_{I}\right) g}{\rho_{I}} \frac{h_{I}+s x}{h_{I} s x}}\left[J_{1}(X) \cos T+\left\{J_{0}(X)-\frac{J_{1}(X)}{X}\right\} \sin T\right]
\end{aligned}
$$

where $A$ is a constant; and $J_{p}$ is the Bessel function of order $p$. The dependent variables are given by

$$
X=\frac{2 \sigma}{s} \sqrt{\frac{\rho_{I}}{\left(\rho_{I I}-\rho_{I}\right) g} \frac{h_{I} s x}{h_{I}+s x}} \text {, and } T=-\sigma t
$$

For convenience, the dependent variable $X$ will not be shown hereafter. A displacement of the interface will cause an associated surface displacement. In the upper layer, the linearized momentum equation may be simply given by

$$
\frac{\partial u_{I}}{\partial t}=-g \frac{\partial \eta_{I}}{\partial x}
$$

The continuity equation can be determined in the same manner:

$$
\begin{aligned}
u_{I} & =\frac{C\left(\eta_{I}-\eta_{I I}\right)}{h_{I}+\eta_{I}-\eta_{I I}} \approx-\frac{C}{h_{I}} \eta_{I I} \\
& =\frac{A^{2} C}{2} \frac{h_{I}+s x}{h_{I}^{2} s x}\left[J_{1}(X) \cos T+\left\{J_{0}(X)-\frac{J_{1}(X)}{X}\right\} \sin T\right]^{2}-\frac{A C}{h_{I}}\left\{J_{0}(X) \sin T+J_{1}(X) \cos T\right\}
\end{aligned}
$$

Combining Eqs.(8) and (9), the following differential results: 


$$
\begin{aligned}
\eta_{I}= & -\frac{A^{2} \sigma C}{g} \int \frac{h_{I}+s x}{h_{I}^{2} s x}\left[J_{1} \sin T-\left\{J_{0}-\frac{J_{1}}{X}\right\} \cos T\right]\left[J_{1} \cos T+\left\{J_{0}-\frac{J_{1}}{X}\right\} \sin T\right] d t \\
& -\frac{A \sigma C}{g h_{I}} \int\left\{J_{0} \cos T-J_{1} \sin T\right\} d t
\end{aligned}
$$

\section{Theoretical Water Particle Trajectory}

In the case of internal waves on a slope, we denote $\left(x_{I}(t), z_{I}(t)\right)$ and $\left(x_{I I}(t), z_{I I}(t)\right)$ the instantaneous water particle positions at the time $t$ in the upper and lower layers, respectively. The corresponding horizontal velocities become $u_{I}=\partial x_{I} / \partial t, w_{I}=\partial z_{I} / \partial t, u_{I I}=\partial x_{I I} / \partial t$, and $w_{I I}=\partial z_{I I} / \partial t$. When the mean position of a water particle is given at $\left(\overline{x_{I}}, \overline{z_{I}}\right)$ for the upper layer or at $\left(\overline{x_{I I}}, \overline{z_{I I}}\right)$ for the lower layer, the instantaneous water particle position is denoted as $x_{I}(t)=\overline{x_{I}}+\varsigma_{I}(t)$ and $z_{I}(t)=\overline{z_{I}}+\xi_{I}(t)$, or $x_{I I}(t)=\overline{x_{I I}}+\varsigma_{I I}(t)$ and $z_{I I}(t)=\overline{z_{I I}}+\xi_{I I}(t)$, where $\varsigma_{I}, \xi_{I}$ and $\varsigma_{I I}, \xi_{I I}$ are the horizontal and vertical displacements in the upper layer and lower layer, respectively. When the internal wave is assumed to be the long wave, the vertical displacements in both layers are zero; i.e. $\xi_{I}(t)=0$ and $\xi_{I I}(t)=0$. Thus, expansion of velocities in the Taylor series to the second order and integration of those horizontal velocities with respect to time yield

$$
\begin{aligned}
& \varsigma_{I}=\int u_{I}\left(x_{I}+\varsigma_{I}, z_{I}+\xi_{I}\right) d t=\int\left\{\left(u_{I}\right)_{\overline{x_{I}}, \overline{z_{I}}}+\varsigma_{I}\left(\frac{\partial u_{I}}{\partial x}\right)_{\overline{x_{I}}, \overline{z_{I}}}+\cdots\right\} d t \\
& \varsigma_{I I}=\int u_{I I}\left(x_{I I}+\varsigma_{I I}, z_{I I}+\xi_{I I}\right) d t=\int\left\{\left(u_{I I}\right)_{\overline{x_{I I}}, \overline{z_{I I}}}+\varsigma_{I I}\left(\frac{\partial u_{I I}}{\partial x}\right)_{\overline{x_{I}}, \overline{z_{I I}}}+\cdots\right\} d t
\end{aligned}
$$

Substituting Eqs.(7) and (9) into Eqs.(11) and (12) and manipulating them, the horizontal displacements are given by

$$
\begin{aligned}
\varsigma_{I}= & \frac{A^{2} C}{2 \sigma h_{I}} \frac{h_{I}+s x}{h_{I} s x}\left\{-J_{1}^{2}\left(\frac{T}{2}+\frac{\sin 2 T}{4}\right)+J_{1} J_{01} \frac{\cos 2 T}{2}+J_{01}^{2}\left(-\frac{T}{2}+\frac{\sin 2 T}{4}\right)\right\}-\frac{A C}{\sigma h_{I}}\left(J_{0} \cos T-J_{1} \sin T\right) \\
\varsigma_{I I}= & -\frac{A}{\sigma} \sqrt{\frac{\left(\rho_{I I}-\rho_{I}\right) g}{\rho_{I}} \frac{h_{I}+s x}{h_{I} s x}}\left(J_{1} \sin T+J_{01} \cos T\right) \\
& +\frac{A^{2}}{\sigma^{2}} \frac{\left(\rho_{I I}-\rho_{I}\right) g}{\rho_{I}} \frac{h_{I}+s x}{h_{I} s x}\left[\frac{1}{2 s x^{2}} \frac{h_{I} s x}{h_{I}+s x}\left(J_{1}^{2} \frac{\cos 2 T}{4}+J_{1} J_{01} \frac{\sin 2 T}{2}-J_{01}^{2} \frac{\cos 2 T}{4}\right)\right. \\
& -J_{1} J_{01} \frac{d X}{d x} \frac{\cos 2 T}{4}+J_{0}\left\{J_{1} \frac{d X}{d x}-\frac{1}{X}\left(\frac{1}{2 s x^{2}} \frac{h_{1} s x}{h_{1}+s x} J_{1}-J_{01} \frac{d X}{d x}\right)\right\}\left(-\frac{T}{2}+\frac{\sin 2 T}{4}\right) \\
& \left.-J_{01}^{2} \frac{d X}{d x}\left(\frac{T}{2}+\frac{\sin 2 T}{4}\right)-J_{01}\left\{J_{1} \frac{d X}{d x}-\frac{1}{X}\left(\frac{1}{2 s x^{2}} \frac{h_{1} s x}{h_{1}+s x} J_{01} \frac{d X}{d x}\right) \frac{\cos 2 T}{4 \sigma}\right\}\right]
\end{aligned}
$$

where $J_{01}=J_{0}-J_{1} / X$.

\section{Radiation stress, setup, and attenuation for internal waves}

Umeyama \& Shintani (2006) applied the radiation stress concept (Longuet-Higgins and Stewart 1964) to calculate the setup or set-down of internal waves. The principal component of the radiation stress for internal waves was given by

$$
S_{x x}=\overline{\overline{\eta_{I}+h_{I}}\left(p_{I}+\rho_{I} u_{I}^{2}\right) d z}-\int_{0}^{h_{I}} p_{I 0} d z+\overline{\int_{-h_{I I}}^{\eta_{I I}}\left(p_{I I}+\rho_{I I} u_{I I}^{2}\right) d z}-\int_{-h_{I I}}^{0} p_{I I} d z
$$


where $S_{x x}$ is the radiation stress for internal waves; $p_{I 0}$ is the hydrostatic pressure in the upper layer; $p_{I I 0}$ is the hydrostatic pressure in the lower layer; and the over-bar denotes averaging in time over a wave period. On the analogy of the surface waves, Eq.(15) can be rearranged as flows :

$$
S_{x x} \approx \int_{0}^{h_{I}} \rho_{I} \overline{\left(u_{I}^{2}-w_{I}^{2}\right)} d z+\int_{-h_{I I}}^{0} \rho_{I I} \overline{\left(u_{I I}^{2}-w_{I I}^{2}\right)} d z
$$

where $w_{I}$ and $w_{I I}$ are the vertical velocities in the upper and lower layers. Umeyama (2002) expressed the first-order horizontal and vertical velocity components as

$$
\begin{aligned}
& u_{I}=\frac{a k}{\sigma}\left(\alpha \cosh k z-\frac{\sigma^{2}}{k} \sinh k z\right) \cos (k x-\sigma t) \\
& w_{I}=-\frac{a k}{\sigma}\left(\alpha \sinh k z-\frac{\sigma^{2}}{k} \cosh k z\right) \sin (k x-\sigma t) \\
& u_{I I}=-\frac{a \sigma}{\sinh k h_{I I}} \cosh k\left(z+h_{I I}\right) \cos (k x-\sigma t) \\
& w_{I I}=\frac{a \sigma}{\sinh k h_{I I}} \sinh k\left(z+h_{I I}\right) \sin (k x-\sigma t)
\end{aligned}
$$

where $a$ is the amplitude of internal waves; $k$ is the wave number; and $\alpha$ is a constant that is given by

$$
\alpha=\frac{\rho_{I I}-\rho_{I}}{\rho_{I}} g-\frac{\rho_{I I}}{\rho_{I}} \frac{\sigma^{2}}{k} \operatorname{coth} k h_{I I}
$$

Substituting Eqs.(17)-(20) into Eq.(16) and integrating the result, the radiation stress yields

$$
S_{x x}=\frac{a^{2}}{2}\left[\rho_{I}\left(\frac{k^{2} h_{I}}{\sigma^{2}} \alpha^{2}-\sigma^{2} h_{I}\right)+\frac{\rho_{I I} \sigma^{2} h_{I I}}{\sinh ^{2} k h_{I I}}\right]
$$

The change in radiation stress leads to a change in the mean level of the density interface when internal waves encounter a sloping beach. The equilibrium between radiation stress change and average slope of the density interface yields

$$
\frac{d S_{x x}}{d x}-\left\{\rho_{I}-\rho_{I I}\right\} g\left(\bar{\eta}+h_{I I}\right) \frac{d \bar{\eta}}{d x}=0
$$

Generally, the attenuation of internal waves in the continental slope is a complicated hydrodynamic process. For the purpose of the present study it may be helpful to use a model in which the divergence of the energy flux is balanced by the dissipation. Therefore, the equation of energy conservation can be expressed as (Umeyama \& Shintani 2006)

$$
\frac{d F}{d x}=-f_{w} \frac{\rho_{I}+\rho_{I I}}{3 \pi}\left(\frac{\sigma a}{\sinh k h_{I I}}\right)^{3}-\beta \frac{\left(\rho_{I}+\rho_{I I}\right) g}{4 \pi \sqrt{2}} \sigma a^{2}
$$

where $F$ is the energy flux per unit of width; $f_{w}$ is the friction coefficient; and $\beta$ is the energy dissipation coefficient.

\section{EXPERIMENTS}

Experiments were carried out in an internal-wave tank of $6.0 \mathrm{~m}$ long, $0.15 \mathrm{~m}$ wide, and $0.35 \mathrm{~m}$ deep (see Figure 1). A slide-type wavemaker with a D-shaped paddle and a linear actuator was placed at one end of the tank, and a 1-cm-thick Plexiglas plate with a slope of 3 in 50 was placed between 100 and $600 \mathrm{~cm}$ from the wavemaker-side wall. The test section was located in the area from $\mathrm{x}=20 \mathrm{~cm}$ to 195 


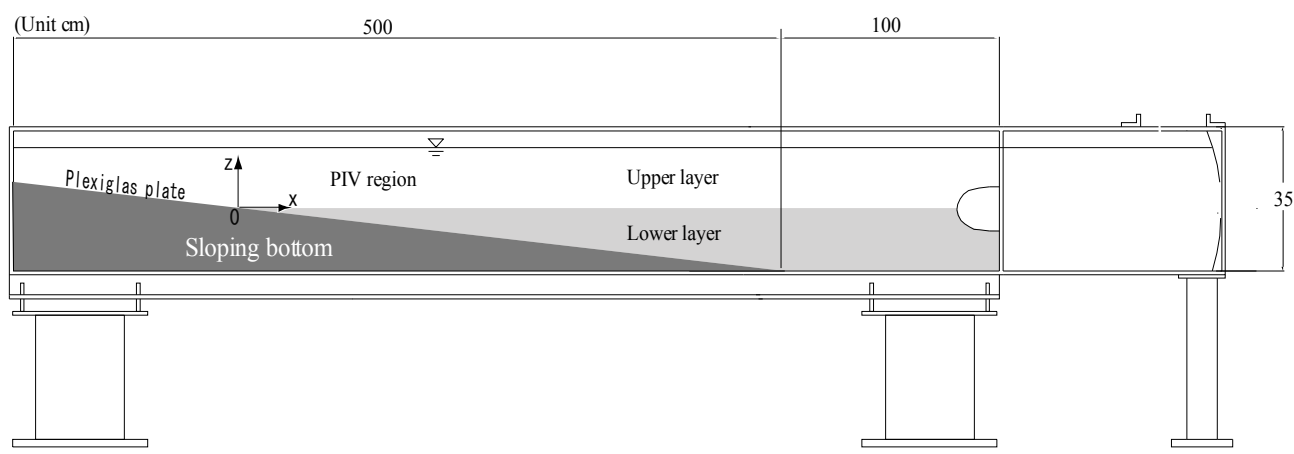

Figure 1. Sketch of experiments

\begin{tabular}{|c|c|c|c|c|c|}
\hline \multicolumn{6}{|c|}{ "Table 1. Experimental cases } \\
\hline CASE & Slope & Density & $\mathrm{h}_{\mathrm{I}}: \mathrm{h}_{\mathrm{II}}$ & $\begin{array}{c}\text { Wave } \\
\text { height* }\end{array}$ & $\begin{array}{l}\text { Wave } \\
\text { period }\end{array}$ \\
\hline 1 & \multirow{2}{*}{$3 / 50$} & \multirow{2}{*}{$1,030 \mathrm{mg} / \mathrm{cm}^{3}$} & \multirow{2}{*}{$12 \mathrm{~cm}: 18 \mathrm{~cm}$} & $2.22 \mathrm{~cm}$ & $5.2 \mathrm{~s}$ \\
\hline 2 & & & & $2.10 \mathrm{~cm}$ & $7.2 \mathrm{~s}$ \\
\hline 3 & \multirow{2}{*}{$3 / 50$} & \multirow{2}{*}{$1,030 \mathrm{mg} / \mathrm{cm}^{3}$} & \multirow{2}{*}{$15 \mathrm{~cm}: 15 \mathrm{~cm}$} & $2.60 \mathrm{~cm}$ & $5.2 \mathrm{~s}$ \\
\hline 4 & & & & $2.70 \mathrm{~cm}$ & $7.2 \mathrm{~s}$ \\
\hline
\end{tabular}

* Wave height was measured using wave gauge at $100 \mathrm{~cm}$ from the wavemaker.

cm downstream of the paddle. A density-stratified fluid consisting of fresh water and salt water was prepared for the experiments. The density of the salt water was $1,030 \mathrm{mg} / \mathrm{cm}^{3}$. The total water depth from the bottom of the wave tank was kept at $30 \mathrm{~cm}$. A summary of the experimental conditions is presented in Table 1.

The instantaneous water particle velocity was measured in two continuous areas of $91.0 \mathrm{~cm}$ long and $27.0 \mathrm{~cm}$ high (one glass panel) using PIV consisting of Nd:YAG laser of 8-W energy. A 2-mm light sheet was emitted from the upper side: this light sheet had very uniform intensity and was well suited to the PIV measurement in a large area. The system included two high-definition digital video cameras (SONY HXR-NX5J) with a maximum resolution of $1920 \times 1080$ pixels. These video cameras were arranged in a line approximately $1 \mathrm{~m}$ from the sidewall of the wave tank. The water in the tank was seeded with DIAION (DK-FINE HP20), having a grain size of $0.25 \mathrm{~mm}$ and specific gravity of 1.01 . The interrogation window was $12 \times 12$ pixels, while the candidate region was $24 \times 24$ pixels. Vector fields were obtained by processing a pair of image frames. The PIV algorithm was based on the crosscorrelation method. The resultant displacement of topographic features for two image frames spaced at $\Delta t=0.1 \mathrm{~s}$ was chosen for direct calculation of the velocity vectors. After the PIV measurement that gave the velocities available at spatially discrete nodal locations in a Eulerian scheme, we estimated each individual particle velocity and location. For a complete discussion of the procedure, refer to Umeyama et al. (2010)

A flow visualization technique was also used to quantify the interfacial displacement. In the experiment, a blue dye was added to the water in the lower layer to aid visualization of the density field. The halogen lamps were lying on the floor at one side of the wave tank, and two video cameras were positioned at the other side. The method of segmentation was used to detect the interfacial displacement. The measured displacements gave the wave celerity and wave setup along the slope.

\section{RESULTS}

\section{Interfacial displacements}

Figure 2 shows the spatial displacements of the density interface for Cases 1, 2, 3, and 4. The abscissa is the distance from the intersection between the sloping bed and stationary level of the density interface, and the ordinate is the elevation above that level. The circular symbols show the density interface by using the image processing technique. The solid curve shows the analytical solution based on Eq.(6). The experimental data points for Cases 1 and 2 are missing due to the flume flange in the range of $10 \mathrm{~cm}<\mathrm{x}<30 \mathrm{~cm}$. Inspecting these figures reveals that the difference between the theoretical 

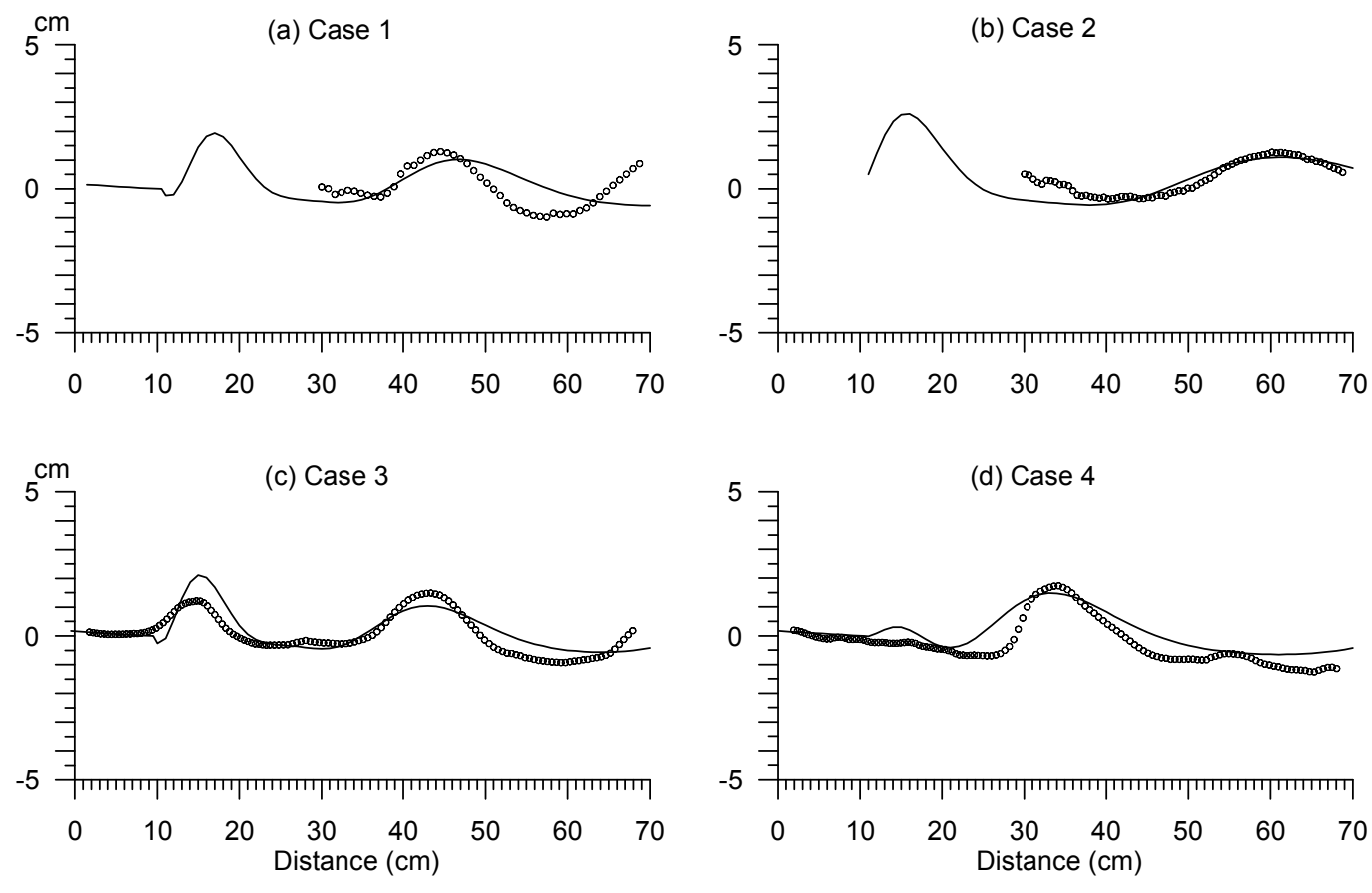

$\circ \quad$ Measured

Method of characteristics

Figure 2. Spatial displacements of density interface

and measured profiles is large for Case 1 but small for Case 2. Generally, the test data agree well with the theoretical interfacial displacement as the location approaches the origin because of the assumption given in the section of theory. For Case 3, the experimental waveform in the front wave is flatter but that in the rear wave is peeked near the crest, when comparing it to the theoretical profile. It is clear that the original pattern of waveform varies owing to the wave breaking and interaction with the backflow from the upper slope, but the theoretical curve by the method of characteristics predicts the measured profile with a reasonable degree of accuracy in the total range. The details of the internal-wave propagation in the experiment of Case 4 are relatively well represented in the theoretical solution with small error. The theoretical solution slightly overshoots the measured density interface: the experimental wave might evolve from a theoretical profile due to the backflow effect.

\section{Instantaneous velocity fields}

The internal waves were generated from the wavemaker located in one side of wave tank. The wave height was measured at $100 \mathrm{~cm}$ from it using an internal wave gage. When a group of internal waves propagates shoreward, oscillatory water particle movements are induced in both upper and lower layers. Figure 3 shows the velocity vector fields due to such internal waves for Case 2. Figures 3 (a) and (b) depict velocity vector distributions from $\mathrm{x}=20 \mathrm{~cm}$ to $110 \mathrm{~cm}$ and $\mathrm{x}=120 \mathrm{~cm}$ to $195 \mathrm{~cm}$, respectively. The measured instantaneous velocity fields were depicted for four different phase values of $t / T=0,0.25$, 0.50 , and 0.75 . The abscissa and ordinate are the horizontal and vertical distances from the origin, respectively. Internal waves propagate from right to left, i.e., in the negative $\mathrm{x}$ direction. The corresponding wave height is $\mathrm{h}=2.22 \mathrm{~cm}$ at $\mathrm{x}=100 \mathrm{~cm}$. Figure 3 (a) depicts the velocity fields for the measured region that is closer to the origin. The results show that the size of vortices gradually decreases when internal waves travel from offshore to inshore. The change of vortices involves the energy dissipation effect due to the instabilities and mixing during the runup event on a slope. As a result, vortices become thinner but velocities increase toward the upper slope. Inspecting the velocity in the region from $\mathrm{x}=40 \mathrm{~cm}$ to $60 \mathrm{~cm}$ reveals that the vortices changes its shape from elliptic shape to round shape when it transfers cross this region. It means that the internal wave may break due to the enhancement of velocity in this region. After the internal wave breaks, it generates a bolus of dense fluid and propagates up the slope. The round shape of vortices near $\mathrm{x}=50 \mathrm{~cm}$ for the phase of $\mathrm{t} / \mathrm{T}=0.0$ proves this tendency. In other phase values of $\mathrm{t} / \mathrm{T}=0.25,0.5,0.75$, this bolus propagates and decreases 
(a)
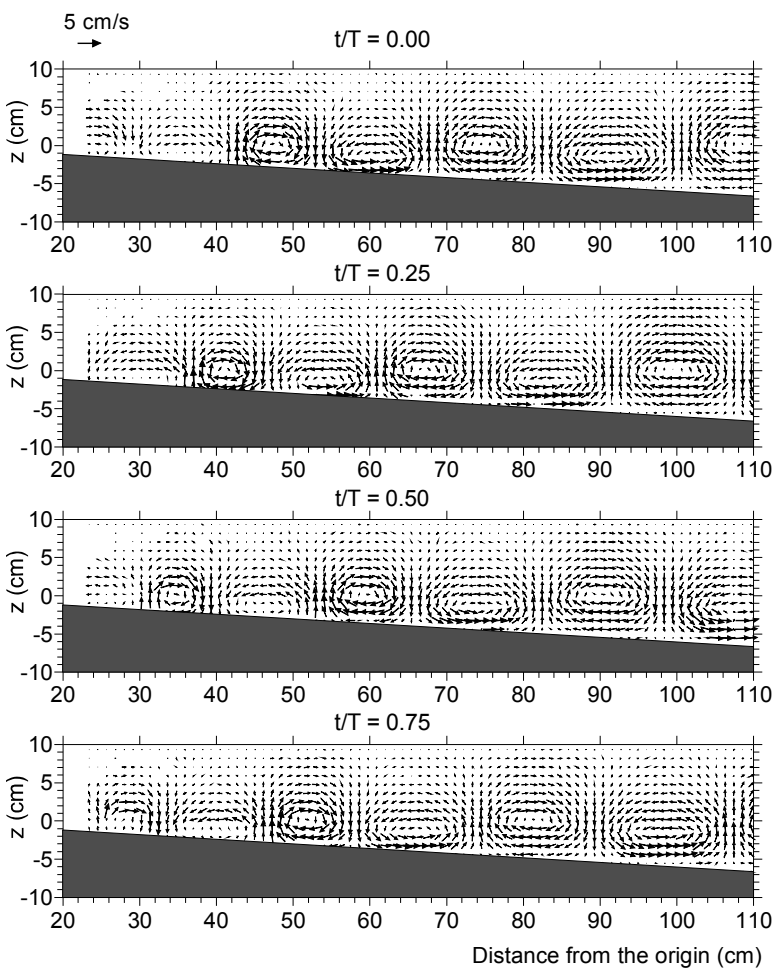

(b)
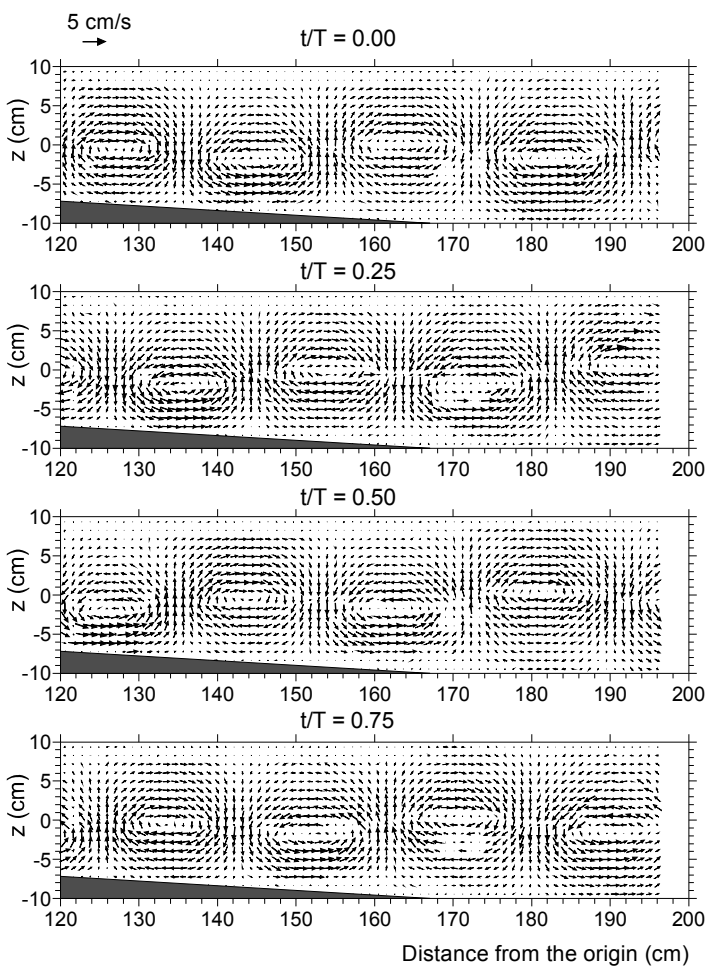

Figure 3. Velocity fields for Case 1

(a)
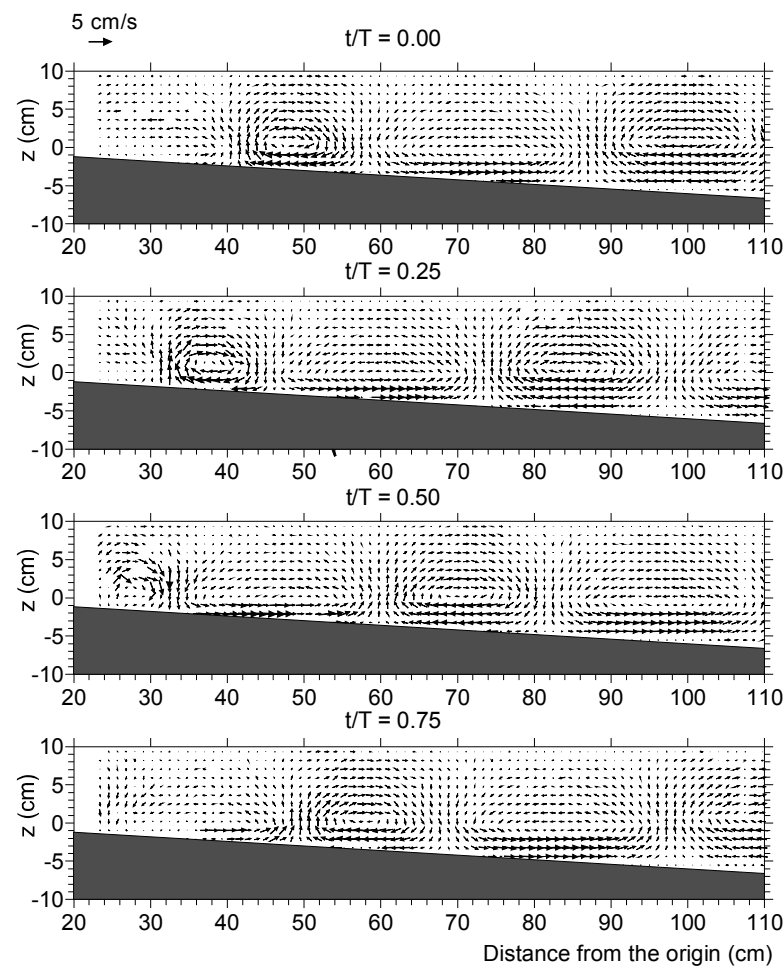

(b)
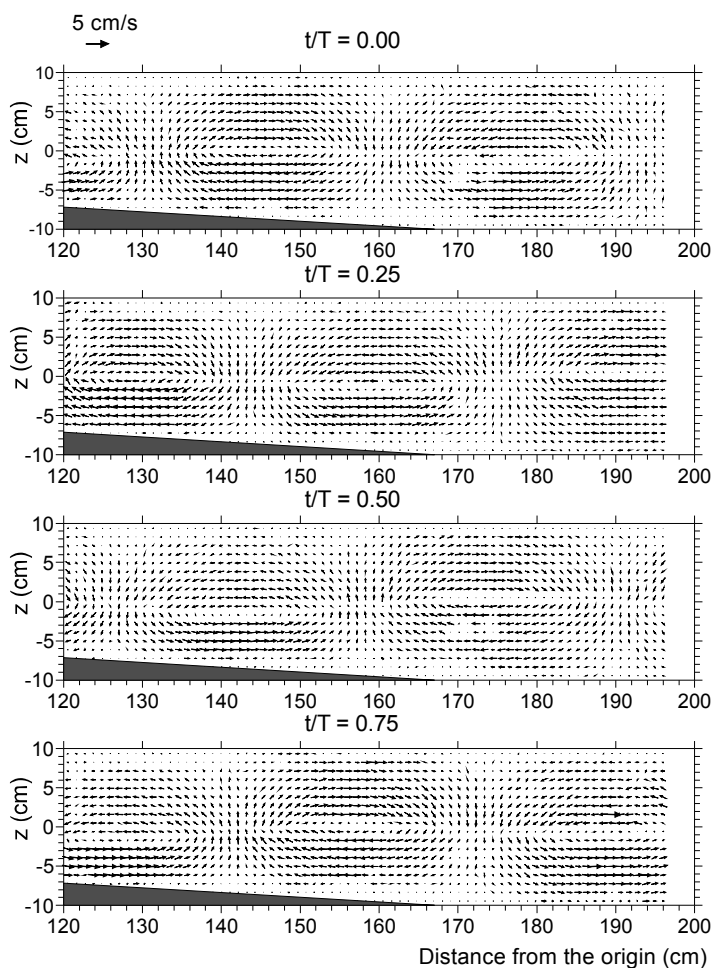

Figure 4. Velocity fields for Case 2 
its size and speed due to the interaction with the backflow. In Figure 3 (b), there is an imposing array of asymmetric vortices, in which a thicker clockwise vortex alternates with a depressed counterclockwise vortex. The scales of these vortices decrease when the waves propagate along a slope. Near the density interface, the mean velocity in the lower layer appears to be relatively larger than that in the upper layer. As a consequence, the pairs of vortices have been distorted but they still keep the quasi-elliptical shape. In addition, the center of ellipse departs upward from the mean density interface at the wave crest, while it deviates downward slightly from the interface at the wave trough. The particle velocity reaches its maximum near the density interface. The flow converges in the front of the wave crest and diverges behind it.

Figure 4 illustrates the measured velocity fields for one wave cycle with an interval of T/4 in the period of $\mathrm{T}=7.2 \mathrm{~s}$ for Case 2 . The clockwise and counterclockwise vortices are in an orderly line when compared with those in the period of $\mathrm{T}=5.2 \mathrm{~s}$. This fact suggests that an increase of wave period leads to an increase in the stability of vortices that gradually decrease the size with distance up the slope. From a pair of counterrotating vortices, one can expect the nonlinearity of internal wave, although the difference of two vertical positions for the counterrotating vortices is relatively small. It seems that the vortex becomes more flat when it propagates to the shallow water before breaking and becomes round trap of dense water.

\section{Water particle trajectory}

Figure 5 shows the plots of the measured particle orbit geometries for Case 1 during three wave cycles of $\mathrm{T}=5.2 \mathrm{~s}$ at three locations: (a) $\mathrm{x}=60 \mathrm{~cm}$, (b) $\mathrm{x}=90 \mathrm{~cm}$, and (c) $\mathrm{x}=190 \mathrm{~cm}$. The triangle symbol shows a position of water particle estimated from the PIV measurement at intervals of $2 \mathrm{~cm}$. At $\mathrm{x}=60 \mathrm{~cm}$, the water particles near the interface drift toward to shore while the other particles in the remaining layers tend to move in the direction opposing to the wave propagation to conserve mass in whole flume. Water particles move clockwise in the upper layer and anticlockwise in the lower layer. This tendency is quite similar at $\mathrm{x}=90 \mathrm{~cm}$. Figure 5 (c) shows the different movement of water particle very close to the interface at deeper region $(\mathrm{x}=190 \mathrm{~cm})$. The particle near the density interface skips with the wave propagation. It seems that the particle moves further when compared with that in the other location. When the internal waves propagate in the two-layer stratified fluid over a constant slope, the excursion of particle near the interface becomes shorter due to the decrease of the wave speed caused by the dissipation and friction. Waves generate upslope-surging vortex cores of dense fluid (boluses) after wave breaking and these cores continue to propagate further up the slope. This fact explains why the particles near the density interface in the upper layer drift relatively long distance toward the shore.

Figure 6 depicts the water particle trajectories for the same thickness ratio but the different wave period $(\mathrm{T}=7.2 \mathrm{~s})$. The initial positions of water particle are $\mathrm{x}=60 \mathrm{~cm}, 90 \mathrm{~cm}$ and $190 \mathrm{~cm}$. Careful inspection of the oscillations of water particles in all elevations confirms that the particle trajectories are not closed in the total depth. At $\mathrm{x}=60 \mathrm{~cm}$, the particle moves similar to that in the period of $\mathrm{T}=5.2 \mathrm{~s}$ except the excursion length. The water particles near the density interface tend to move in the direction of the wave propagation while the other particles in the remaining region travel in the opposite direction. Near the bottom, the water particle oscillations are quite identical at three locations during three wave cycles.

Figure 7 shows the theoretical trajectory calculated for Cases 1 and 2 by Eqs.(13) and (14) whose higher terms lead to an asymmetry of the particle oscillation. In these calculations, the vertical positions were calculated using Eq.(10) that gives the displacement of the density interface. Note that ideal trajectory data cannot be obtained without removing extraneous effects such as reflection from the wall and higher harmonics generated by the wavemaker, although the size of the wave tank restricts these effects. From the present comparisons for internal waves, however, the experimental path is qualitatively in good agreement with the theoretical trajectory just above and below the density interface. 
(a) $x=60 \mathrm{~cm}$

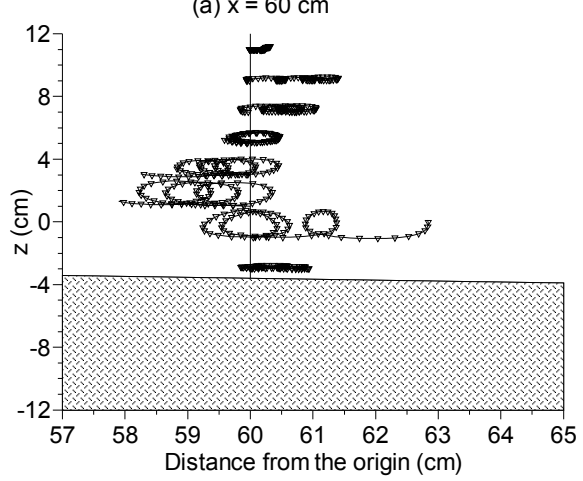

(b) $x=90 \mathrm{~cm}$

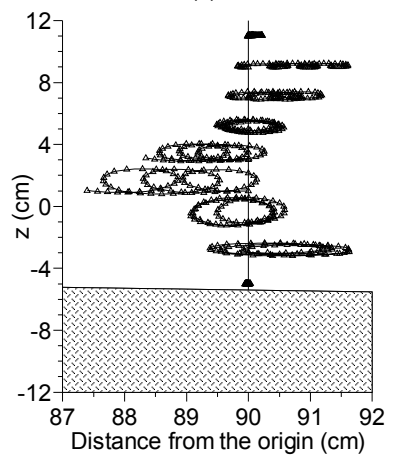

(c) $x=190 \mathrm{~cm}$

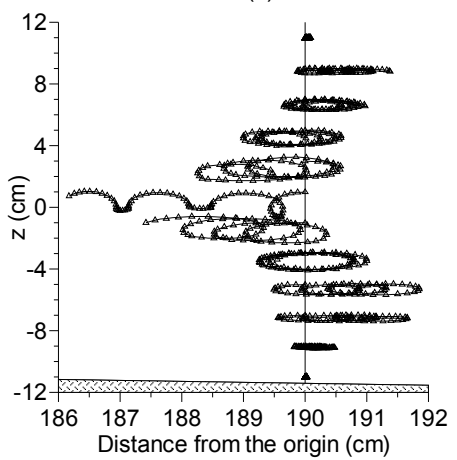

Figure 5. Water particle trajectories for Case 1

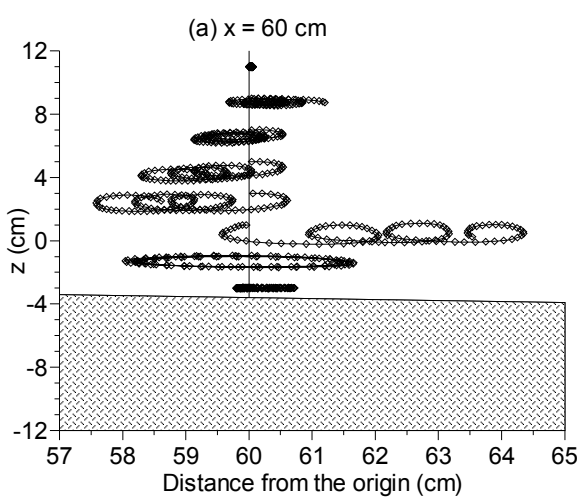

(b) $x=90 \mathrm{~cm}$

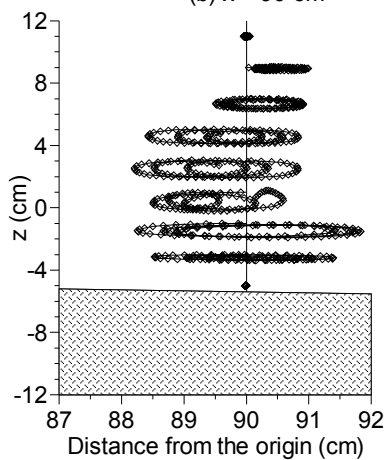

(c) $x=190 \mathrm{~cm}$

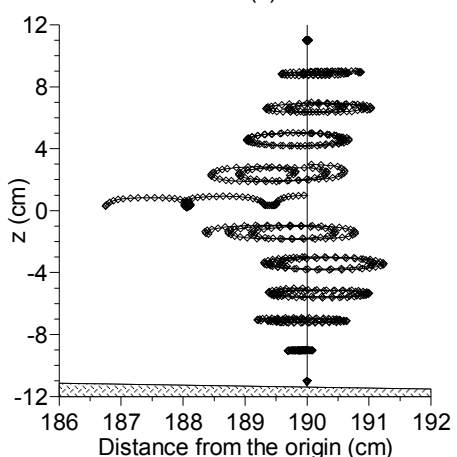

Figure 6. Water particle trajectories for Case 2

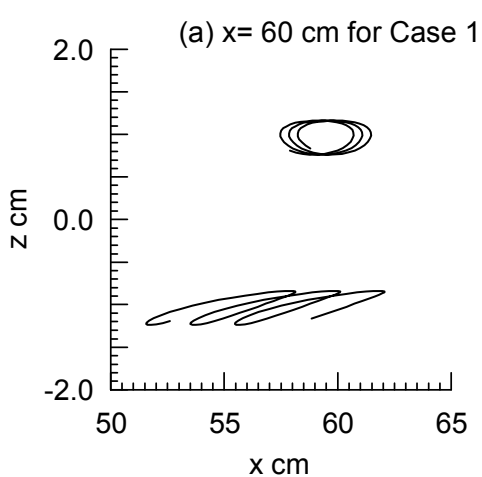

(d) $\mathrm{x}=60 \mathrm{~cm}$ for Case 2

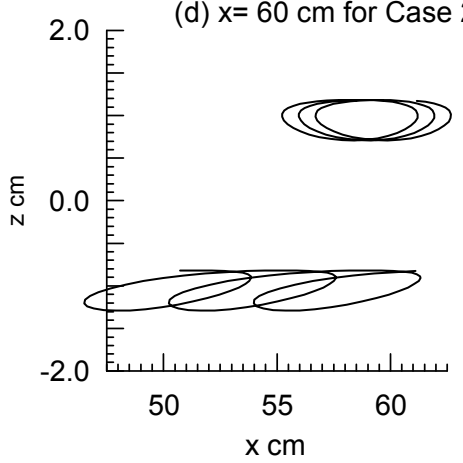

(b) $\mathrm{x}=90 \mathrm{~cm}$ for Case 1

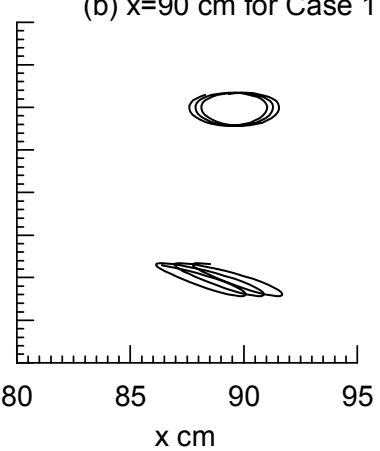

(e) $\mathrm{x}=90 \mathrm{~cm}$ for Case 2

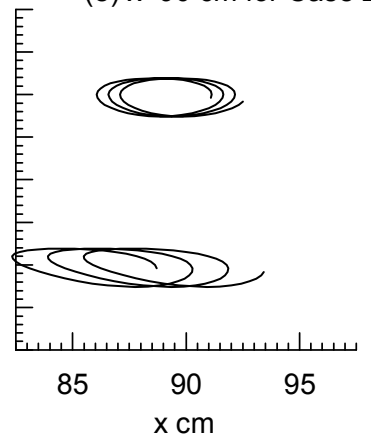

(c) $\mathrm{x}=190 \mathrm{~cm}$ for Case 1

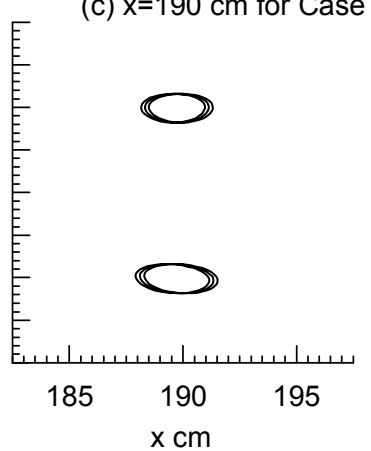

(f) $\mathrm{x}=190 \mathrm{~cm}$ for Case 2

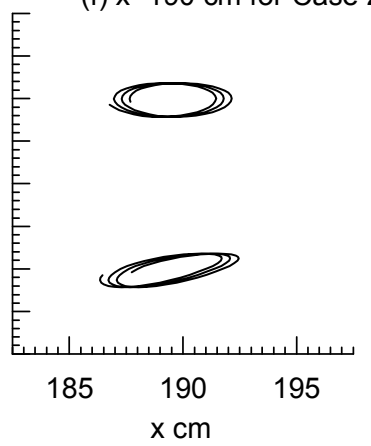

Figure 7. Calculated particle paths by the Method of characteristics for Cases 1 and 2 
Internal wave celerity
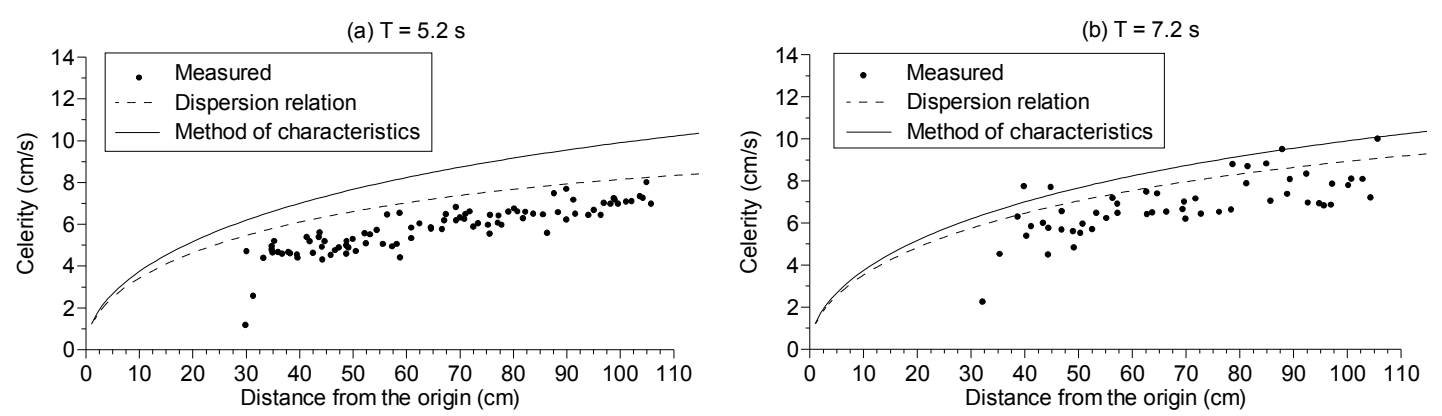

Figure 8. Distributions of wave celerity for Cases 1 and 2

In addition to the distributions of velocity vectors and particle trajectories along the gentle slope, we considered spatial variations of wave celerity. Lamb (1932) proposed a dispersion relation between wave speed and wavenumber from the linearized theory as

$c=\sqrt{\frac{g}{k} \frac{\rho_{I I}-\rho_{I}}{\rho_{I} \operatorname{coth} k h_{I}+\rho_{I I} \cosh k h_{I I}}}$

The wave celerity was estimated from spatial wave profiles obtained by image processing technique. Figure 8 (a) and (b) show the variations of internal wave celerity for Cases 1 and 2, respectively. The measured and theoretical celerity variations are depicted in the same figure. The tendency of measured data is in good agreement with the theoretical distributions by the dispersion relation and the method of characteristics. The internal wave celerity tends to decrease toward the origin. For the period of $\mathrm{T}=5.2 \mathrm{~s}$, the measured celerity is always smaller than those estimated from the dispersion relation and the method of characteristics. We attribute the difference between the measured and theoretical celerity profiles to the bottom topography. Eq.(24) was derived for a flat bottom condition, but the present bottom of wave tank was consisting of a sloping bottom and a deeper constant depth. Figure 8 (b) shows the similar comparison of the celerity profiles for the period of $\mathrm{T}=7.2 \mathrm{~s}$. It is noticed that for this case the measured celerity reasonably well fits with theoretical one when compared with that for the period of $\mathrm{T}=5.2 \mathrm{~s}$. It suggests that only numerical method may work well for calculations for this type of topography, but additional components such as frictions in the formula of dispersion relation and the method of characteristics will expand a range of computation.

\section{Attenuation and setup for internal waves}

Figure 9 shows comparisons of predicted and observed variations of the local wave height and the mean density interface for Cases 3 and 4. Illustrated in the solid curve are the wave transformation predicted using Eq.(23) and the wave setup or set-down using Eq.(22). A portion of the incident wave motion is converted to the forward translation of the water mass by the breaking process. This results in forming a bolus that runs further up the face of the slope. The maximum runup elevation depends on the wave steepness and the bottom angle. Although the reflection coefficient should be determined in advance of the calculations of attenuation and setup, the reflection was neglected in this study. In order to calculate the wave-height distribution, the value of $\beta$ in Eq.(23) was determined using a trial-anderror procedure. In the present computation, it was equal to 0.020 for Case 3 and 0.025 for Case 4 . For shoaling waves on a given bottom slope, the wave height decreases gradually with decreasing water depth. Good agreement is found between the measured and calculated values of wave height in the recorded region. The discrepancy between these measured and calculated distributions is probably due to the neglect of the reflection or the overestimation of shear stress parameters. The radiation stress for partial standing waves differs from that for incident waves; it is presented by the sum of the incident and reflected waves. For surface waves, Longuet-Higgins and Stewart (1964) found that the mean surface level for a standing wave train slightly increases at the antinodes and correspondingly decreases at the nodes, having twice the frequency of an incident wave train. A somewhat similar relationship was found in the experimental result by Umeyama (2008) for partial standing internal waves. Partial standing internal waves develop a longitudinal mean interfacial oscillation that is a half period out of phase. Although it is difficult to detect these oscillations from the observation data, generally, the theoretical mean interfacial displacement agrees with the measured setup. 

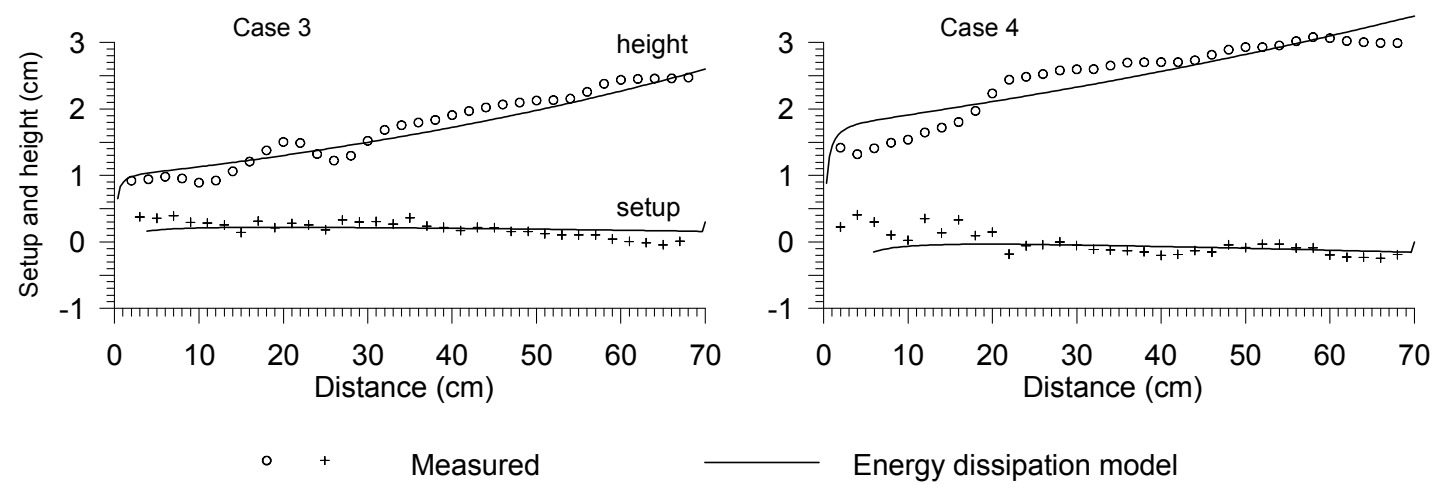

Figure 9. Attenuation and setup of internal waves for Cases 3 and 4

\section{CONCLUSIONS}

In the present investigation, experiments of internal waves propagating over a constant slope have been carried out with a PIV method to illustrate the velocity fields and water particle trajectories in layers of fresh and salt water. Wave celerity of internal waves was investigated theoretically and experimentally. The method of characteristics was adapted to express the interfacial displacements of two fluids of slightly different densities on a sloped topography. The instantaneous velocity vector map clearly illustrated a vortex pair when the thickness ratio was relatively large in a fluid of two density layers. The particle trajectories in a cross-section were simulated using a solution based on the definition of the Lagrangian approach to the method of characteristics. When internal waves propagate up a slope, the particles near the density interface move in the shore direction while those in the lower and upper layers move in the offshore direction.

\section{REFERENCES}

Adrian, R.J. 1991. Particle imaging techniques for experimental fluid mechanics, Annual Review of Fluid Mech., 23, 261-304.

Cacchione, D. A., and C. Wunsch. 1974. Experimental study of internal waves over a slope, Journal of Fluid Mechanics, 66, 223-329.

Carrier, G. F., and Greenspan, H. P. 1957. Water waves of finite amplitude on a sloping beach, Journal of Fluid Mechanics, 4, 97-109.

De Silva, I.P.D., Imberger, J., and G.N. Ivey. 1997. Localized mixing due to a breaking internal wave ray at a sloping bed, Journal of Fluid Mechanics, 350, 1-27.

Grue, F., Jensen, A., Rusas, P.-O. and Sveen, J.K. 1999. Properties of large-amplitude internal waves. Journal of Fluid Mechanics, 380, 257-278.

Helfrich, K. R. 1992. Internal solitary wave breaking and run-up on a uniform slope. Jounal of Fluid Mechanics, 243, 133-154.

Lamb, H. (1932). Hydrodynamics, Dover, pp. 370-372.

Longuet-Higgins, M. S., and Stewart, R. W. 1964. Radiation stress in water waves: A physical discussion, with applications. Deep-Sea Research, 11, 529-562.

Michallet, H., and Ivey G. N. 1999. Experiments on mixing due to internal solitary waves breaking on uniform slopes. Journal of Geophysical Research, 104(C6), 13467-13477.

Shimizu, R., Shintani, T. and Umeyama, M. (2005). Instantaneous and Lagragian velocity fields of internal waves on a slope by PIV measurement and numerical simulation. Annual Journal of Coastal Engineering, 52, 1-5.

Stevens, C. L., and M. J. Coates. 1994. Applications of a maximized cross-correlation technique for resolving velocity fields in laboratory experiments, Journal of Hydraulic Research, IAHR, 32(2), 195-212.

Umeyama, M. 2002. Experimental and theoretical analyses of internal waves of finite amplitude, Journal of Waterway, Port, Coastal and Ocean Engineering, ASCE, 128(3), 133-141.

Umeyama, M. 2008. PIV techniques for velocity fields of internal waves over a slowly varying bottom topography, Journal of Waterway, Port, Coastal and Ocean Engineering, ASCE, 134(5), 286-298. 
Umeyama, M. 2010. Coupled PIV and PTV measurements of particle velocities and trajectories for surface waves following a steady current, Journal of Waterway, Port, Coastal and Ocean Engineering, ASCE, 137(2), 85-94.

Umeyama, M., and H. Matsuki. 2011. Measurements of velocity and trajectory of water particle for internal waves in two density layers, Geophysical Research Letters, 38, AGU, L03612.

Umeyama, M., and H. Shinomiya. 2009. Particle image velocimetry measurements for Stokes progressive internal waves, Geophysical Research Letters, 36(6), AGU, L06603.

Umeyama, M. and Shintani, T. 2004. Visualization analysis of runup and mixing of internal waves on an upper slope, Journal of Waterway, Port, Coastal and Ocean Engineering, ASCE, 130(2), 89-97.

Umeyama, M. and Shintani, T. 2006. Transformation, attenuation, setup, and undertow of internal waves on a gentle slope, Journal of Waterway, Port, Coastal and Ocean Engineering, ASCE, 132(6), 477-486.

Umeyama, M., Shintani, T. \& Watanabe, S., 2010. Measurements of particle velocities and trajectories in a wave-current motion using PIV and PTV, Proceedings of the $32^{\text {nd }}$ International Conference on Coastal Engineering, ASCE, Waves.2.

Wunsch, C. 1971. Note on some Reynolds stress effects of internal waves on slopes, Deep-Sea Research, 18, 583-591.

Willert, C.E. and M. Gharib. 1991. Digital particle image velocimetry, Experiments in Fluids, 10, 181193. 